\title{
Consumo de substâncias psicoativas pelos estudantes de medicina e sua relação com o programa de mentoria
}

\section{Consumption of psychoactive substances among medicine students and its relationship to mentoring program}

\author{
Isabelle Salomão Teixeira Silva1. Thais de Paula Silva Pilio¹. Andrea Frozino Ribeiro ${ }^{1}$. \\ 1 Pontifícia Universidade Católica de Minas Gerais, Betim, Minas Gerais, Brasil.
}

\section{RESUMO}

Objetivos: Identificar o padrão de uso das substâncias psicoativas, os fatores associados a esse uso, e a percepção do programa de mentoria diante do comportamento de risco de estudantes do curso de medicina da Pontifícia Universidade Católica de Minas Gerais. Metodologia: Foi realizado um estudo descritivo, observacional e transversal por meio da disponibilização de um formulário on-line contendo questões sobre as informações sociodemográficas, o perfil de uso de substâncias de abuso (moderado, de risco ou dependente) e a percepção do programa de mentoria aos estudantes de medicina. A coleta de dados foi realizada entre fevereiro a novembro de 2019. Resultados: Maior consumo de risco foi observado entre os usuários de álcool, tabaco e maconha. Os estudantes no regime de internato apresentaram maior abuso de álcool. Dentre os fatores de risco destaca-se a presença de um parente com consumo abusivo e o início do consumo antes dos 18 anos de idade. O programa de mentoria foi percebido de forma positiva para a prevenção do comportamento de risco, embora a maioria dos que avaliaram apresentavam consumo de risco para o álcool e/ou outras drogas. Conclusão: O consumo abusivo de substâncias pode ser minimizado por meio de ações implementadas nos programas de mentorias.

Palavras-chave: Transtornos relacionados ao uso de substâncias. Drogas ilícitas. Doenças não transmissíveis. Alostase. Tutoria.

\section{ABSTRACT}

Objectives: To identify the pattern of use of psychoactive substances, the factors associated to drug use and the perception of the Mentoring Program and its relation to risk behavior from students of the curse of Medicine at Pontifícia Universidade Católica of Minas Gerais. Methodology: A descriptive, observational, and transverse study was made using an online form for collecting sociodemographic information, pattern of use of drug abuse (moderate, risk, or dependent), and perception of the Mentoring Program from medical students. The data collect was carried out between February and November, 2019. Results: Higher risk consumption was observed among alcohol, tobacco and marijuana users. Students at the boarding school experienced greater alcohol abuse. Among the risk factors, the presence of a relative with abusive consumption and the beginning of consumption before the age of 18 stand out. Although the mentoring program was evaluated positively for the prevention of risky behavior, the majority of those who evaluated it presented risk consumption for alcohol and/or other drugs. Conclusion: More actions can be implemented within the mentoring in order to minimize the harmful impacts of drug abuse.

Keywords: Substance-related disorder. Illicit drugs. Noncommunicable diseases. Allostasis. Mentoring.

Autor correspondente: Andrea Frozino Ribeiro, Rua do Rosário, 1081, Bairro Angola, Betim, Minas Gerais, Brasil. CEP: 60450-060. Telefone: +55 31 3319-4444. E-mail: andreafroz@pucminas.br

Conflito de interesses: Não há qualquer conflito de interesses por parte de qualquer um dos autores.

Recebido em: 07 Set 2020; Revisado em: 18 Abr 2021; Aceito em: 02 Jul 2021. 


\section{INTRODUÇÃO}

A busca pelo equilíbrio consiste em uma meta para a população humana, equilíbrio esse que nos permite manter uma saúde mental e física em bom estado. Contudo, os problemas psiquiátricos se encontram entre os que mais prejudicam a saúde da humanidade há mais de duas décadas, sendo o Transtorno do uso de substâncias (Substance use disorder, SUD) um dos mais representativos, conforme o relatório Global Burden Disease (2016).

O consumo abusivo de álcool é um relevante problema de saúde pública, acarretando mais de 3 milhões de mortes em 2016 e contribuindo para a manifestação de mais de 200 tipos de doenças, transtornos mentais e danos à saúde em todo o mundo. ${ }^{1}$ No Brasil, $17,9 \%$ da população adulta ( $\geq 18$ anos) faz uso abusivo de bebidas alcoólicas, sendo que esse número aumentou $14,7 \%$ em relação ao levantamento realizado em 2006. ${ }^{2}$ Entre universitários, o consumo de álcool e outras drogas de abuso é elevado quando comparado à população geral. ${ }^{3,4}$ Além disso, também são observados acidentes automobilísticos, comportamento sexual de risco, violência, menor interesse pelos estudos pelos universitários que abusam das substâncias. ${ }^{5,6}$

Diversos modelos do programa de mentoria são descritos variando os objetivos, período de implementação e participação voluntária ou obrigatória, mas com a finalidade em comum de auxiliar aos estudantes em suas experiências profissionais e pessoais. ${ }^{7,8,9}$ Dessa forma, para identificar o padrão de uso (moderado, de risco ou dependente) das substâncias psicoativas, os fatores associados a esse uso e a percepção do programa de mentoria diante do comportamento de risco, foi conduzida uma pesquisa por meio de questionário disponibilizado aos estudantes do curso de medicina da Pontifícia Universidade Católica de Minas Gerais (PUCMG).

\section{MATERIAIS E MÉTODOS}

Amostra: foi realizado um estudo descritivo, observacional, com corte transversal, em estudantes matriculados na PUC-MG no curso de Medicina de todos os períodos. Os critérios de inclusão foram estar matriculado no curso de graduação de medicina, campus Betim, independente do período, e ter idade superior a 18 anos. O critério de exclusão foi estar matriculado em outro curso de graduação. Todos os procedimentos foram aprovados pelo Comitê de Ética em Pesquisa da PUC-MG sob o número 91833118.6.0000.5137/2018.

Triagem dos voluntários: os voluntários foram contatados por meio de e-mail registrado na PUC-MG e pelos grupos acadêmicos de cada período do curso em mídia social (i.e., Whatsapp). O questionário foi disponibilizado por meio de formulário digital on-line elaborado no Google Forms, durante o período de fevereiro de 2019 a novembro de 2019 e, o número de respostas foi restrito a uma vez. Foram enviados 752 e-mails para todos os estudantes do curso e
20 mensagens padronizadas via mídia social com diferentes grupos de estudantes, com o intervalo de quatro meses entre as duas. O questionário foi respondido por 65 estudantes, dois foram excluídos por não estarem matriculados no curso de interesse.

Questionários: o formulário continha um roteiro estruturado com 48 questões acerca de dados sociodemográficos ${ }^{10}$ (idade, sexo biológico, ciclo de estudo, auxílio financeiro, classe social, religião), uso das substâncias de abuso ${ }^{11,12,13}$ (AUDIT, ASSIST, relação parental com usuário de álcool e/ou outras drogas de abuso, idade de início do uso do álcool e/ou outras drogas de abuso, percepção sobre os transtornos do uso do álcool) e avaliação da tutoria acadêmica ${ }^{14,15}$ (percepção do papel do mentor, frequência dos encontros, percepção do relacionamento com o mentor, percepção sobre o resultado da mentoria). Breve descrição do AUDIT (Alcohol Use Disorders Identification Test), utilizado para detectar o consumo de risco no último ano, por meio de uma escala que varia entre 0 a 40 pontos. Entre 0 a 7 pontos (zona I) estão os usuários abstêmios ou com consumo de baixo risco. Entre 8 a 15 pontos (zona II), os usuários apresentam um consumo de risco e a intervenção deve ser realizada por meio de orientação básica. Entre 16 a 19 pontos (zona III), os usuários apresentam um consumo nocivo com problemas de saúde em decorrência do abuso de álcool podendo estar com o Transtorno de uso de álcool (TUA ou AUD - Alcohol use disorder) conhecido como abuso (DSM-V: 305.00; CID-10: F10.10) e nessa condição a intervenção breve (IB) é requerida. Acima de 20 pontos (zona IV) aumenta a probabilidade de os usuários estarem com o TUA conhecido como dependência (DSM-V: 303.90; CID-10: F10.20) e devem ser encaminhados a uma avaliação especializada.

Breve descrição do ASSIST (Alcohol Smoking And Substance Involvement Screening Test), detecta problemas relacionados ao uso de álcool, tabaco, maconha, cocaína, estimulantes (anfetamina), inalantes, ansiolíticos/sedativos/hipnóticos, alucinógenos, opiáceos entre outras nos últimos três meses. A pontuação varia entre 0 a 39 pontos a depender do tipo de droga utilizada. Acima de 10 pontos para o álcool e 3 pontos para as demais drogas, os usuários necessitam de IB da mesma maneira que os usuários da zona III do AUDIT. Já acima de 26 pontos é necessário o encaminhamento para o tratamento especializado, similarmente ao que ocorre aos usuários que caem na zona IV do AUDIT.

Análise estatística: os períodos de estudo foram divididos em ciclo básico ( $1^{\circ}$ ao $4^{\circ}$ ano), ciclo clínico ( $5^{\circ}$ ao $8^{\circ}$ ano) e ciclo internato $\left(9^{\circ}\right.$ ao $12^{\circ}$ ano). As variáveis categoriais foram apresentadas em relação à sua frequência (contagem e porcentagem) e analisadas por meio do teste de $X^{2}(n>10)$ ou $V^{2}(n<10)$. Para a análise de correlação foi utilizado o teste de Spearman. O limite de confiança estipulado foi de $95 \%$ e o nível de significância mínimo menor que 0,05 . Foi utilizado o programa STATISTICA 13.5 (TIBCO Software Inc., São Caetano do Sul). 


\section{RESULTADOS}

\section{Características sociodemográficas}

Aproximadamente $8,5 \% \quad(n=63)$ do total de discentes responderam ao questionário. A amostra foi estratificada considerando as faixas etárias entre 18 a 22 anos $(n=29$, $46 \%), 23$ a 27 anos $(n=23,36 \%), 28$ a 32 anos ( $n=7,11 \%)$, e 33 a 37 anos $(n=4,6 \%)$. A maioria era composta por mulheres $\left(n_{\text {mulheres }}=46,73 \% ; n_{\text {homens }}=17,27 \%\right)$. Considerando os ciclos de estudo quase metade da amostra estava no básico $\left(n_{\text {básico }}=\right.$ $30,48 \% ; n_{\text {clínico }}=19,30 \%$ e $n_{\text {internato }}=14,22 \%$ ).

Cerca de um terço da amostra declarou ser beneficiário de algum tipo de auxílio financeiro para pagar os estudos ( $n=19,30 \%$ ), [i.e., FIES, PROUNI ou bolsas sociais da própria Instituição de Ensino Superior (IES)] e o restante não $(n=44,69 \%)$. As classes sociais elevadas, i.e, A e B, representaram $63 \%$ dos estudantes (A: $n=17,27 \%$; B1: $n=$ $12,19 \%$; B2: $n=11,17 \%$; C1: $n=9,14 \%$; $2: n=10,16 \%$; e DE: $n=4,6 \%$ ). A maioria dos participantes declararam ter uma religião e não foi observada diferença $(p>0,05)$ com relação a religião e comportamento de risco (material suplementar).

\section{Comportamento de risco diante das substâncias psicoativas}

Para o AUDIT, a maioria dos estudantes apresentou um consumo de baixo risco $(n=54 ; 85,7 \%)$ e o restante um consumo de risco $(n=9 ; 14,3 \%)$. Dentre os que apresentaram consumo de risco, a maioria é do sexo feminino $(n=6 ; 66,7 \%)$, está nas altas classes sociais $(n=8 ; 88,9 \%)$ e no ciclo do internato $\left(n=5 ; 55,5 \% ; X^{2}=6,75, p=0,009\right)$. Quanto maior o tempo de curso maior a pontuação no AUDIT $(r=0,35, p<$ $0,05)$.

Para o ASSIST foram considerados válidos 61 questionários e a droga que possui o maior número de estudantes necessitando de IB era o álcool $\left(n_{\text {álcool }}=7 ; 11,5 \% ; n_{\text {tabaco ou maconha }}=5 ; 8,2 \%\right.$; $\left.n_{\text {estimulantes }}=3 ; 4,9 \% ; n_{\text {sedativos }}=2 ; 3,3 \%\right)$. Um dos usuários de hipnóticos/sedativos também apresentou consumo de risco para o álcool. Na modalidade outras drogas não houve necessidade de quaisquer intervenções. Considerando o uso de drogas por injeção somente um estudante relatou ter feito uso, mas não nos últimos 3 meses $(n=1 ; 1,6 \%)$ e um estudante optou por não responder $(n=1 ; 1,6 \%)$. Dentre os que precisam de IB para todas as drogas, a maioria é do sexo feminino $(n=12 ; 85,7 \%)$, estão nas altas classes sociais $(n=$ $11 ; 78,6 \%)$ e no ciclo básico $(n=6 ; 42,9 \%)$. Considerando apenas o álcool cerca de $57,1 \%(n=4)$ era do ciclo clínico ou internato.

\section{Relação parental}

Um pouco mais da metade dos participantes $(n=34 ; 54 \%)$ relatou que possui um familiar que bebe em excesso ou é dependente de álcool, sendo que o restante respondeu que não $(n=24 ; 38,1 \%)$ ou não sabe $(n=5 ; 7,9 \%)$. A maioria $\operatorname{dos}$ consumidores de risco relatou apresentar um familiar com problemas relacionados ao uso do álcool $(n=6 ; 66,7 \%)$.
Acerca do uso de drogas de abuso, a situação se inverte, sendo que mais da metade relatou não ter um familiar que faz uso de drogas $(n=33 ; 54,1 \%)$ e, o restante possui $(n=15$; $24,6 \%)$ ou não sabe $(n=13 ; 21,3 \%)$. Dentre os usuários que necessitam de IB para o uso de drogas e/ou álcool, 35,7\% $(n=5)$ declararam ter um familiar que usa drogas e $64,3 \%$ $(n=9)$ declararam que não ou não sabem. Contudo, quando consideramos apenas os usuários de drogas que necessitam de IB (sem o álcool), 55,5\% $(n=5)$ têm algum familiar que é usuário $(n=5,55,5 \%)$.

\section{Primeiro uso do álcool elou outras drogas}

Com relação a idade do primeiro consumo de álcool, um pouco menos da metade dos participantes fez seu primeiro uso dos 16 aos 20 anos $(n=30 ; 47,6 \%)$, em seguida dos 11 aos 15 anos $(n=26 ; 41,3 \%)$, depois um pequeno número dos 21 aos 25 anos $(n=3 ; 4,8 \%)$, e por último os dois extremos: dos 5 aos 10 anos ou dos 26 aos 30 anos $(n=1 ; 1,6 \%$ /cada). Alguns participantes optaram por não responder $(n=2,3,1 \%)$. Dentre os consumidores de risco, a maioria $(n=8,89 \%)$ experimentou o álcool abaixo dos 18 anos e um entre 21 e 25 anos.

Quanto ao primeiro contato com o uso de drogas, mais da metade dos estudantes não responderam $(n=33 ; 54,1 \%)$. Considerando a faixa etária de primeiro uso da droga foi observado maior consumo dos 16 aos 20 anos $(n=15 ; 24,6 \%)$, seguido dos 21 aos 25 anos $(n=9 ; 14,7 \%), 11$ aos 15 anos $(n=$ $7 ; 11,5 \%)$ e somente um participante dos 26 aos 30 anos $(n=$ $1 ; 1,6 \%)$. Dentre os que precisavam de IB, mais da metade não respondeu a idade de início de uso da droga $(n=5 ; 55,5 \%)$ e $35,7 \%(n=5)$ fez uso antes dos 18 anos de idade.

\section{Percepção do programa de Mentoria}

O mentor era melhor percebido como referência de atitudes, orientador, professor e conselheiro (Tabela 1 e 1S). A periodicidade dos encontros de maior prevalência era mensal (Tabela 2 e 2S). As ações do mentor melhor percebidas eram as relacionadas ao desenvolvimento profissional (Tabela 3 e 3S). Os principais desfechos percebidos pelos estudantes considerando a interação com o mentor foram os relacionados à formação pessoal (melhor relação com os colegas de classe, automotivação, autocontrole, ânimo, bom-humor, sensação de bem-estar, redução do estresse e desenvolvimento pessoal) e não profissional (Tabela 4 e 4 S). Cerca de $66,7 \%$ dos estudantes afirmaram que o programa de mentoria ajuda a evitar o comportamento de risco $(n=42)$, enquanto $33,3 \%$ negaram $(n=21)$. A maioria dos que apresentaram consumo de risco (escala AUDIT; $n=5 ; 55,5 \%$ ) e com necessidade de IB (escala ASSIST; $n=9 ; 64,3 \%$ ), percebeu o programa de mentoria de forma útil na prevenção de comportamento de risco. Para os com consumo de risco, 44,4\% $(n=4)$ concordaram que a relação com o mentor ajudava a evitar riscos na saúde física e/ou psíquica caindo para 33,3\% $(n=3)$ considerando as situações de risco que ameaçasse o avanço da carreira. Dentre os com necessidade de IB o resultado foi similar, sendo que $42,9 \%(n=6)$ concordaram que a relação com o mentor prevenia riscos na saúde e 24,4\% $(n=3)$ concordaram que prevenia situações de risco que prejudicassem a carreira. 
Tabela 1. Percepção dos estudantes sobre o papel do mentor considerando as áreas de atuação $(n=63)$.

\begin{tabular}{|c|c|c|c|c|c|c|c|c|}
\hline Área & Papel do mentor & DT & $\mathrm{D}$ & $\mathrm{DP}$ & $\mathrm{CP}$ & $\mathrm{C}$ & $\mathrm{CT}$ & NS \\
\hline \multirow[t]{4}{*}{ Profissional } & Professor & $5(7,9 \%)$ & $5(7,9 \%)$ & $1(1,6 \%)$ & $8(12,7 \%)$ & $20(31,7 \%)$ & $23(36,5 \%)$ & $1(1,6 \%)$ \\
\hline & Conselheiro & $4(6,3 \%)$ & $3(4,8 \%)$ & $2(3,2 \%)$ & $7(11,1 \%)$ & $25(39,7 \%)$ & $22(34,9 \%)$ & $0(0 \%)$ \\
\hline & Orientador & $3(4,8 \%)$ & $2(3,2 \%)$ & $2(3,2 \%)$ & $7(11,1 \%)$ & $24(38,1 \%)$ & $25(39,7 \%)$ & $0(0 \%)$ \\
\hline & Provedor de ideias & $5(7,9 \%)$ & $0(0 \%)$ & $5(7,9 \%)$ & $11(17,5 \%)$ & $23(36,5 \%)$ & $19(30,1 \%)$ & $0(0 \%)$ \\
\hline \multirow[t]{3}{*}{ Pessoal } & Defensor & $5(7,9 \%)$ & $3(4,8 \%)$ & $11(17,5 \%)$ & $11(17,5 \%)$ & $17(27 \%)$ & $14(22,2 \%)$ & $2(3,2 \%)$ \\
\hline & Padrinho & $7(11,1 \%)$ & $6(9,5 \%)$ & $5(7,9 \%)$ & $13(20,6 \%)$ & $12(19 \%)$ & $16(25,4 \%)$ & $4(6,3 \%)$ \\
\hline & Agente para contato & $3(4,8 \%)$ & $5(7,9 \%)$ & $6(9,5 \%)$ & $7(11,1 \%)$ & $19(30,1 \%)$ & $21(33,3 \%)$ & $2(3,2 \%)$ \\
\hline Ambas & $\begin{array}{l}\text { Referência de atitudes, } \\
\text { comportamentos e } \\
\text { valores }\end{array}$ & $5(7,9 \%)$ & $4(6,3 \%)$ & $7(11,1 \%)$ & $3(4,8 \%)$ & $16(25,4 \%)$ & $28(44,4 \%)$ & $0(0 \%)$ \\
\hline
\end{tabular}

Dados $n(\%)$ de uma escala Likert (DT: discordo totalmente; D: discordo; DP: discordo parcialmente; CP: concordo parcialmente; C: concordo; CT concordo totalmente; NS: não se aplica).

Tabela 2. Frequência dos encontros entre mentor e estudantes $(n=63 *)$.

\begin{tabular}{|c|c|c|c|c|c|c|c|}
\hline Frequência & DT & $\mathrm{D}$ & DP & $\mathrm{CP}$ & $\mathrm{C}$ & $\mathrm{CT}$ & NS \\
\hline Diariamente & $47(74,6 \%)$ & $3(4,8 \%)$ & $1(1,6 \%)$ & $0(0 \%)$ & $0(0 \%)$ & $1(1,6 \%)$ & $10(15,9 \%)$ \\
\hline Semanalmente & $41(65,1 \%)$ & $3(4,8 \%)$ & $1(1,6 \%)$ & $3(4,8 \%)$ & $2(3,2 \%)$ & $3(4,8 \%)$ & $9(14,3 \%)$ \\
\hline Mensalmente & $12(19 \%)$ & $1(1,6 \%)$ & $2(3,2 \%)$ & $4(6,3 \%)$ & $16(25,4 \%)$ & $25(39,7 \%)$ & $3(4,7 \%)$ \\
\hline Bimestralmente & $31(49,2 \%)$ & $5(7,9 \%)$ & $2(3,2 \%)$ & $1(1,6 \%)$ & $6(9,5 \%)$ & $7(11,1 \%)$ & $9(14,3 \%)$ \\
\hline Semestralmente & $31(49,2 \%)$ & $6(9,5 \%)$ & $0(0 \%)$ & $2(3,2 \%)$ & $5(7,9 \%)$ & $7(11,1 \%)$ & $10(15,9 \%)$ \\
\hline
\end{tabular}

Dados $n(\%)$ de uma escala Likert (DT: discordo totalmente; D: discordo; DP: discordo parcialmente; CP: concordo parcialmente; C: concordo; CT concordo totalmente; NS: não se aplica). ${ }^{*}$ Sem resposta: Diariamente e semanalmente $[1$ (1,6\%)]; bimestralmente e semestralmente $[2$ $(3,2 \%)]$.

Tabela 3. Percepção das habilidades inter-relacionais do mentor para com os estudantes $(n=63)$.

\begin{tabular}{|c|c|c|c|c|c|c|c|c|}
\hline $\begin{array}{l}\text { Habilidades/ } \\
\text { Conhecimentos }\end{array}$ & Relacionamento & DT & $\mathrm{D}$ & DP & $\mathrm{CP}$ & $\mathrm{C}$ & $\mathrm{CT}$ & NS \\
\hline \multirow[t]{4}{*}{$\begin{array}{l}\text { Conhecimento } \\
\text { técnico }\end{array}$} & Meu mentor é acessível. & $3(4,8 \%)$ & $2(3,2 \%)$ & $1(1,6 \%)$ & $8(12,7 \%)$ & $15(23,8 \%)$ & $31(49,2 \%)$ & $3(4,8 \%)$ \\
\hline & $\begin{array}{l}\text { Meu mentor demonstra } \\
\text { integridade profissional. }\end{array}$ & $1(1,6 \%)$ & $1(1,6 \%)$ & $2(3,2 \%)$ & $2(3,2 \%)$ & $14(22,2 \%)$ & $39(61,9 \%)$ & $4(6,3 \%)$ \\
\hline & $\begin{array}{l}\text { Meu mentor demonstra } \\
\text { conhecimento em minha } \\
\text { área de interesse. }\end{array}$ & $5(7,9 \%)$ & $3(4,8 \%)$ & $3(4,8 \%)$ & $6(9,5 \%)$ & $18(28,6 \%)$ & $26(41,3 \%)$ & $2(3,2 \%)$ \\
\hline & $\begin{array}{l}\text { Meu mentor é } \\
\text { encorajador e solidário. }\end{array}$ & $3(4,8 \%)$ & $1(1,6 \%)$ & $3(4,8 \%)$ & $3(4,8 \%)$ & $18(28,6 \%)$ & $33(52,4 \%)$ & $2(3,2 \%)$ \\
\hline \multirow[t]{2}{*}{ Comunicação } & $\begin{array}{l}\text { Meu mentor promove } \\
\text { críticas construtivas e } \\
\text { úteis do meu trabalho. }\end{array}$ & $5(7,9 \%)$ & $0(0 \%)$ & $2(3,2 \%)$ & $7(11,1 \%)$ & $25(39,7 \%)$ & $23(36,5 \%)$ & $1(1,6 \%)$ \\
\hline & $\begin{array}{l}\text { Meu mentor me motiva } \\
\text { e melhora o resultado do } \\
\text { meu trabalho. }\end{array}$ & $5(7,9 \%)$ & $0(0 \%)$ & $4(6,3 \%)$ & $8(12,7 \%)$ & $20(31,7 \%)$ & $25(39,7 \%)$ & $1(1,6 \%)$ \\
\hline
\end{tabular}

Dados n (\%) de uma escala Likert (DT: discordo totalmente; D: discordo; DP: discordo parcialmente; CP: concordo parcialmente; C: concordo; CT concordo totalmente; NS: não se aplica).

Continua. 
Conclusão.

Tabela 3. Percepção das habilidades inter-relacionais do mentor para com os estudantes $(n=63)$.

\begin{tabular}{|c|c|c|c|c|c|c|c|c|}
\hline $\begin{array}{l}\text { Habilidades/ } \\
\text { Conhecimentos }\end{array}$ & Relacionamento & DT & D & DP & $\mathrm{CP}$ & $\mathrm{C}$ & CT & NS \\
\hline \multirow[t]{5}{*}{ Educador } & $\begin{array}{l}\text { Meu mentor é útil em } \\
\text { direcionar e guiar as } \\
\text { questões profissionais. }\end{array}$ & $3(4,8 \%)$ & $2(3,2 \%)$ & $4(6,3 \%)$ & $9(14,3 \%)$ & $19(30,1 \%)$ & $25(39,7 \%)$ & $1(1,6 \%)$ \\
\hline & $\begin{array}{l}\text { Meu mentor me responde } \\
\text { satisfatoriamente (com } \\
\text { clareza, compreensão, } \\
\text { agilidade). }\end{array}$ & $3(4,8 \%)$ & $2(3,2 \%)$ & $3(4,8 \%)$ & $7(11,1 \%)$ & $26(41,3 \%)$ & $20(31,7 \%)$ & $2(3,2 \%)$ \\
\hline & $\begin{array}{l}\text { Meu mentor apoia as } \\
\text { minhas contribuições } \\
\text { apropriadamente. }\end{array}$ & $4(6,3 \%)$ & $3(4,8 \%)$ & $1(1,6 \%)$ & $6(9,5 \%)$ & $24(38,1 \%)$ & $22(34,9 \%)$ & $3(4,8 \%)$ \\
\hline & $\begin{array}{l}\text { Meu mentor me provém } \\
\text { de forma apropriada } \\
\text { (fontes de materiais, } \\
\text { contatos) }\end{array}$ & $6(9,5 \%)$ & $1(1,6 \%)$ & $5(7,9 \%)$ & $11(17,5 \%)$ & $22(34,9 \%)$ & $16(25,4 \%)$ & $2(3,2 \%)$ \\
\hline & $\begin{array}{l}\text { Meu mentor me desafia } \\
\text { a ampliar minhas } \\
\text { habilidades (tentar novas } \\
\text { atividades, assumir } \\
\text { riscos, escrever um } \\
\text { artigo) }\end{array}$ & $7(11,1 \%)$ & $4(6,3 \%)$ & $5(7,9 \%)$ & $11(17,5 \%)$ & $16(25,4 \%)$ & $19(30,1 \%)$ & $1(1,6 \%)$ \\
\hline
\end{tabular}

Dados n (\%) de uma escala Likert (DT: discordo totalmente; D: discordo; DP: discordo parcialmente; CP: concordo parcialmente; C: concordo; CT concordo totalmente; NS: não se aplica).

Tabela 4. Descrição dos produtos, atividades e/ou sentimentos desenvolvidos pelos estudantes com o apoio do mentor $(n=61 *)$.

\begin{tabular}{|c|c|c|c|c|c|c|c|c|}
\hline Campo & Resultado & DT & $\mathrm{D}$ & DP & $\mathrm{CP}$ & $\mathrm{C}$ & $\mathrm{CT}$ & NS \\
\hline \multirow[t]{13}{*}{ Profissional } & Publicação & $29(46 \%)$ & $7(11,1 \%)$ & $1(1,6 \%)$ & $3(4,8 \%)$ & $3(4,8 \%)$ & $1(1,6 \%)$ & $17(26,7 \%)$ \\
\hline & Apresentação ou pôster & $29(46 \%)$ & $7(11,1 \%)$ & $1(1,6 \%)$ & $3(4,8 \%)$ & $2(3,2 \%)$ & $2(3,2 \%)$ & $17(26,7 \%)$ \\
\hline & $\begin{array}{l}\text { Redação de um trabalho } \\
\text { científico }\end{array}$ & $28(44,4 \%)$ & $6(9,5 \%)$ & $0(0 \%)$ & $5(7,9 \%)$ & $2(3,2 \%)$ & $3(4,8 \%)$ & $17(26,7 \%)$ \\
\hline & Atividades comunitárias & $25(39,7 \%)$ & $7(11,1 \%)$ & $0(0 \%)$ & $3(4,8 \%)$ & $4(6,3 \%)$ & $5(7,9 \%)$ & $17(26,7 \%)$ \\
\hline & Organização de evento & $23(36,5 \%)$ & $4(6,3 \%)$ & $0(0 \%)$ & $8(12,7 \%)$ & $6(9,5 \%)$ & $5(7,9 \%)$ & $15(23,8 \%)$ \\
\hline & $\begin{array}{l}\text { Maior rede de contatos na } \\
\text { faculdade }\end{array}$ & $23(36,5 \%)$ & $5(7,9 \%)$ & $2(3,2 \%)$ & $5(7,9 \%)$ & $11(17,5 \%)$ & $6(9,5 \%)$ & $9(14,3 \%)$ \\
\hline & Melhor nota nas provas & $23(36,5 \%)$ & $8(12,7 \%)$ & $1(1,6 \%)$ & $6(9,5 \%)$ & $8(12,7 \%)$ & $4(6,3 \%)$ & $11(17,5 \%)$ \\
\hline & $\begin{array}{l}\text { Desenvolvimento de } \\
\text { atividades extracurriculares }\end{array}$ & $22(34,9 \%)$ & $5(7,9 \%)$ & $1(1,6 \%)$ & $4(6,3 \%)$ & $14(22,2 \%)$ & $6(9,5 \%)$ & $9(14,3 \%)$ \\
\hline & Organização profissional & $15(23,8 \%)$ & $2(3,2 \%)$ & $2(3,2 \%)$ & $10(15,9 \%)$ & $11(17,5 \%)$ & $15(23,8 \%)$ & $6(9,5 \%)$ \\
\hline & Conhecimento clínico & $14(22,2 \%)$ & $4(6,3 \%)$ & $0(0 \%)$ & $15(23,8 \%)$ & $12(19 \%)$ & $6(9,5 \%)$ & $10(15,9 \%)$ \\
\hline & Conhecimento científico & $16(25,4 \%)$ & $2(3,2 \%)$ & $1(1,6 \%)$ & $14(22,2 \%)$ & $11(17,5 \%)$ & $7(11,1 \%)$ & $10(15,9 \%)$ \\
\hline & Emprego melhor & $21(33,3 \%)$ & $6(9,5 \%)$ & $0(0 \%)$ & $8(12,7 \%)$ & $8(12,7 \%)$ & $3(4,8 \%)$ & $15(23,8 \%)$ \\
\hline & $\begin{array}{l}\text { Proteção contra possíveis } \\
\text { situações de risco que } \\
\text { ameace o avanço da carreira }\end{array}$ & $17(27 \%)$ & $3(4,8 \%)$ & $2(3,2 \%)$ & $6(9,5 \%)$ & $15(23,8 \%)$ & $13(20,6 \%)$ & $5(7,9 \%)$ \\
\hline
\end{tabular}

Dados $n(\%)$ de uma escala Likert (DT: discordo totalmente; D: discordo; DP: discordo parcialmente; CP: concordo parcialmente; C: concordo; CT concordo totalmente; NS: não se aplica). Frequência (n); Percentual (\%). *Sem resposta: [2 (3,2\%)].

Continua. 
Conclusão.

Tabela 4. Descrição dos produtos, atividades e/ou sentimentos desenvolvidos pelos estudantes com o apoio do mentor $\left(n=61^{*}\right)$.

\begin{tabular}{|c|c|c|c|c|c|c|c|c|}
\hline Campo & Resultado & DT & $\mathrm{D}$ & DP & $\mathrm{CP}$ & $\mathrm{C}$ & $\mathrm{CT}$ & NS \\
\hline \multirow[t]{11}{*}{ Pessoal } & Sensação de bem-estar & $11(17,5 \%)$ & $4(6,3 \%)$ & $1(1,6 \%)$ & $7(11,1 \%)$ & $17(27 \%)$ & $18(28,6 \%)$ & $3(4,8 \%)$ \\
\hline & Redução do estresse & $11(17,5 \%)$ & $4(6,3 \%)$ & $1(1,6 \%)$ & $10(15,9 \%)$ & $12(19 \%)$ & $19(30,1 \%)$ & $4(6,3 \%)$ \\
\hline & Desenvolvimento pessoal & $8(12,7 \%)$ & $5(7,9 \%)$ & $2(3,2 \%)$ & $7(11,1 \%)$ & $15(23,8 \%)$ & $20(31,7 \%)$ & $4(6,3 \%)$ \\
\hline & $\begin{array}{l}\text { O conselho do mentor é } \\
\text { sempre a melhor ideia }\end{array}$ & $11(17,5 \%)$ & $7(11,1 \%)$ & $10(15,9 \%)$ & $9(14,3 \%)$ & $11(17,5 \%)$ & $8(12,7 \%)$ & $5(7,9 \%)$ \\
\hline & $\begin{array}{l}\text { Melhor relação com os } \\
\text { colegas de classe }\end{array}$ & $12(19 \%)$ & $1(1,6 \%)$ & $1(1,6 \%)$ & $9(14,3 \%)$ & $15(23,8 \%)$ & $18(28,6 \%)$ & $5(7,9 \%)$ \\
\hline & Autoconhecimento & $10(15,9 \%)$ & $4(6,3 \%)$ & $4(6,3 \%)$ & $9(14,3 \%)$ & $14(22,2 \%)$ & $17(27 \%)$ & $3(4,8 \%)$ \\
\hline & Motivação & $9(14,3 \%)$ & $4(6,3 \%)$ & $2(3,2 \%)$ & $11(17,5 \%)$ & $14(22,2 \%)$ & $18(28,6 \%)$ & $3(4,8 \%)$ \\
\hline & $\begin{array}{l}\text { Proteção contra possíveis } \\
\text { situações que me exponha a } \\
\text { riscos (em relação a saúde } \\
\text { física e/ou psíquica) }\end{array}$ & $11(17,5 \%)$ & $3(4,8 \%)$ & $1(1,6 \%)$ & $10(15,9 \%)$ & $12(19 \%)$ & $16(25,4 \%)$ & $8(12,7 \%)$ \\
\hline & Bom-humor & $10(15,9 \%)$ & $4(6,3 \%)$ & $2(3,2 \%)$ & $11(17,5 \%)$ & $11(17,5 \%)$ & $20(31,7 \%)$ & $3(4,8 \%)$ \\
\hline & Ânimo & $10(15,9 \%)$ & $4(6,3 \%)$ & $3(4,8 \%)$ & $8(12,7 \%)$ & $12(19 \%)$ & $19(30,1 \%)$ & $5(7,9 \%)$ \\
\hline & Autocontrole & $10(15,9 \%)$ & $6(9,5 \%)$ & $2(3,2 \%)$ & $7(11,1 \%)$ & $11(17,5 \%)$ & $19(30,1 \%)$ & $6(9,5 \%)$ \\
\hline \multirow[t]{2}{*}{ Campo } & Resultado & DT & $\mathrm{D}$ & DP & $\mathrm{CP}$ & $\mathrm{C}$ & $\mathrm{CT}$ & NS \\
\hline & Automotivação & $10(15,9 \%)$ & $4(6,3 \%)$ & $3(4,8 \%)$ & $9(14,3 \%)$ & $11(17,5 \%)$ & $18(28,6 \%)$ & $6(9,5 \%)$ \\
\hline
\end{tabular}

Dados $n(\%)$ de uma escala Likert (DT: discordo totalmente; D: discordo; DP: discordo parcialmente; CP: concordo parcialmente; C: concordo; CT concordo totalmente; NS: não se aplica). Frequência (n); Percentual (\%). *Sem resposta: [2 (3,2\%)].

\section{DISCUSSÃO}

A prevalência do consumo de risco para o álcool e outras drogas (i.e., tabaco, maconha, derivados anfetamínicos e ansiolíticos) nos estudantes de medicina foi similar ao observado em outros estudos ${ }^{16,17}$ e, após o álcool, as drogas mais consumidas foram o tabaco e a maconha. Por sua vez, o programa de mentoria apresenta-se como uma promissora estratégia para reduzir danos e/ou prevenir o surgimento do SUD.

No presente estudo, não foram identificados usuários nas faixas de maior risco (zonas III e IV, AUDIT), porém, em outro estudo realizado em 2011 em Minas Gerais, embora a maioria dos estudantes de medicina estivesse na zona de risco II $(23,3 \%)$, cerca de $1,9 \%$ dos estudantes se encontravam na zona de risco III. ${ }^{5} \mathrm{O}$ consumo de álcool, tabaco, maconha e outras drogas é alto entre universitários brasileiros ${ }^{18,19}$ e mesmo os estudos que evidenciam baixa quantidade consumida, a frequência é elevada (i.e., semanal, 37,7\%). ${ }^{20}$

Maior consumo de álcool foi observado nos ciclos mais avançados do curso de medicina como observado em outro curso, ${ }^{20}$ indicando a presença de fatores predisponentes durante a formação profissional. Contudo, não podemos afirmar quais são os fatores associados a esse quadro, embora seja reconhecida a presença do estresse no ambiente acadêmico ${ }^{21}$ e a relação entre esse e o abuso de álcool. ${ }^{22,23}$ Por outro lado, o abuso de outras drogas foi maior no ciclo básico do curso, talvez por ser uma fase de experimentação como apontado por outro estudo. ${ }^{17}$ Também não podemos descartar a influência do meio e outras características da personalidade, como busca por novidades, que influenciam a tomada de decisão do jovem diante da experimentação das drogas.

Considerando o consumo de ansiolíticos, também há evidências de alto consumo, ${ }^{17}$ mas no presente estudo houve baixa prevalência do uso dos ansiolíticos e anfetamínicos com necessidade de IB. Os dados apontam para uma prevalência elevada do uso do álcool pelos estudantes de medicina, porém, boa parte dos estudos avalia a frequência por si só não evidenciando a relação de risco do uso (abusivo ou dependente).

A idade de início do uso do álcool e/ou drogas e fatores genéticos têm relação com a manifestação do SUD na fase adulta. Embora no Brasil, seja ilegal a venda de bebidas etílicas para menores de 18 anos de idade, foi observado que mais de $40 \%$ dos estudantes consumiram essas substâncias psicoativas pela primeira vez entre 16 a 20 anos ou 11 a 15 anos, similar ao observado em outro estudo. ${ }^{17}$ Porém, a maioria dos estudantes não responderam em qual idade iniciaram o uso de outras drogas de abuso. Considerando os usuários de risco de álcool e/ou drogas, mais da metade declararam ter parente com padrão de consumo abusivo. Juntos esses dados indicam a presença de fatores de risco que também podem estar influenciando no comportamento de risco dos jovens. 
O consumo de álcool tem se tornado equivalente entre mulheres e homens. ${ }^{1}$ No presente estudo foi observado maior consumo em mulheres, embora a nossa amostra tenha sido predominantemente feminina. É importante destacar que o sexo biológico feminino é mais vulnerável aos efeitos nocivos das substâncias psicoativas e isso contribui para maior prejuízo na saúde mental em menor tempo de uso.

Estudantes das classes sociais mais favorecidas foram os que mais consumiam álcool e/ou drogas, como em outros estudos. ${ }^{3,24,25}$ Vale destacar que a maioria dos voluntários da pesquisa compunha os extratos sociais mais elevados, uma vez que o custo da graduação em medicina em uma IES privada é alto. Entretanto, ter mais recurso financeiro não é fator preditivo para o abuso de substâncias psicoativas. O SUD acomete os indivíduos de todos os níveis sociais em todo o mundo. O problema do abuso das substâncias advém de outras vertentes do comportamento humano, que de forma sucinta resvala na constante necessidade em consolidar estados internos de maior "equilíbrio" (contínua alostase mental e química). ${ }^{26,27}$ Considerando as alterações observadas com o uso continuado, inicia-se uma cascata de processos mentais e físico-químicos cerebrais no usuário que aumentam a probabilidade do abuso se tornar adição (i.e., dependência), a depender dos fatores predisponentes biológicos e externos.

Embora a maioria dos estudantes declararem que conhecem sobre os transtornos do uso de substâncias e suas respectivas classificações, praticamente a metade relatou que não estava preparada para identificar os sintomas no paciente (material suplementar). Ainda, a maior parte dos estudantes relataram que não se sentem preparados para orientar um paciente com SUD. De forma similar a outro estudo, o conhecimento dos estudantes foi maior na medida em que progrediam no curso. ${ }^{28}$ Esses resultados podem refletir a subestimação do SUD por parte dos estudantes, uma vez que o consumo também aumentava com o progresso no curso.

Foi interessante notar que os estudantes percebem o programa de mentoria, bem como a relação com o mentor, como uma estratégia e/ou relação que propicia o desenvolvimento profissional. Contudo, quando indagados sobre o resultado do programa na sua formação, não identificaram ganhos relacionados ao desenvolvimento profissional e sim pessoal. $\mathrm{O}$ resultado observado poderia ser consequência da forma como as reuniões são conduzidas pelo mentor, servindo como momentos de interação entre os próprios discentes,

\section{REFERÊNCIAS}

1. World Health Organization. Global status report on alcohol and health 2018. Genebra: WHO; 2018.

2. Bastos FI, Vasconcellos MT, Boni RB, Reis NB, Coutinho CF. III Levantamento Nacional sobre o uso de drogas pela população brasileira. Rio de Janeiro: Fundação Osvaldo Cruz; 2017.

3. Andrade AG, Duarte PC, Oliveira LG. I Levantamento Nacional com debates e reflexões que extrapolam os campos teóricos e clínicos, culminando em um ambiente descontraído, mas desconectado do objetivo do programa de mentoria no que se refere a melhora na formação do estudante. De toda forma, a percepção dos estudantes sobre o programa de mentoria foi positiva, indicando que este está cumprindo com o seu papel, mesmo que de forma limitada. Vale destacar que ambos os aspectos, pessoal e profissional, são importantes na formação do médico e estando alinhadas, o desempenho do estudante torna-se melhor. Embora a maioria dos estudantes tenham afirmado que o programa de mentoria ajude a evitar o comportamento de risco, dois terços desses estão no grupo de risco para o álcool e/ou drogas, indicando uma percepção do programa e/ou uma autopercepção do comportamento de risco errônea. O interesse em abordar o tema abuso de substâncias no programa de mentoria é presente entre os estudantes de medicina. ${ }^{29}$ A mentoria presencial ${ }^{8,9}$ ou por aplicativo, ${ }^{30}$ para auxiliar o futuro profissional do médico no manejo do risco em situação de potencial abuso de substâncias, é útil para a promoção da saúde, podendo assim contribuir para que os estudantes aprendam a reconhecer os próprios limites e deem mais um passo em direção a busca do real sucesso: equilíbrio.

Considerando as limitações do presente estudo, a baixa adesão dos estudantes foi um grande fator limitante, o que pode acarretar em maior viés na análise estatística inferencial dos dados. Além disso, a composição da amostra considerando as variáveis gênero (sexo biológico) e classe social não apresentaram uma distribuição homogênea. A ausência das respostas considerando as informações sobre o uso das outras drogas de abuso também limitou as análises.

\section{CONCLUSÃO}

A prevalência do consumo de risco para o álcool e outras drogas foi similar a observada em outros estudos. Fatores como uso em idade precoce, presença de familiares usuários de álcool e outras drogas e, subestimação do Transtorno do uso de substâncias, podem contribuir para o comportamento de risco diante das substâncias de abuso. Por outro lado, o Programa de mentoria tem o potencial de minimizar e/ou prevenir o abuso de substâncias psicoativas entre os estudantes de medicina.

\section{FINANCIAMENTOS}

A pesquisa não teve fomento para a sua execução, exceto a concessão de bolsas PROUNI, PUC Institucional e FIP 22509-1S. sobre o uso de álcool, tabaco e outras drogas entre universitários das 27 capitais brasileiras. Brasília: SENAD; 2010.

4. Johnston LD, O'Malley PM, Bachman JG, Schulenberg JE. Monitoring the future: national survey results on drug use, 19752009: volume II, college students and adults ages 19-50. Bethesda: National Institution Drug Abuse; 2010.

5. Rocha LA, Lopes AC, Martelli DR, Lima VB, Martelli-Júnior H. 
Consumo de álcool entre estudantes de Faculdades de Medicina de Minas Gerais, Brasil. Rev Bras Educ Med. 2011;35(3):369-75.

6. Carneiro EB, Braga RT, Silva FD, Nogueira MC. Fatores associados a beber pesado episódico entre estudantes de medicina. Rev Bras Educ Med. 2012;36:524-30.

7. Tan YS, Teo SW, Pei Y, Sng JH, Yap HW, Toh YP, et al. A framework for mentoring of medical students: thematic analysis of mentoring programmes between 2000 and 2015. Adv Health Sci Educ Theory Pract. 2018;23(4):671-97.

8. Buddeberg-Fischer B, Herta KD. Formal mentoring programmes for medical students and doctors: a review of the Medline literature. Med Teach. 2006;28(3):248-57.

9. Taherian K, Shekarchian M. Mentoring for doctors: do its benefits outweigh its disadvantages? Med teach. 2008;30:95-9.

10. Associação Brasileira de Empresas de Pesquisa. Critério de classificação econômica Brasil. São Paulo: ABEP; 2008.

11. Mendéz EB. Uma versão brasileira do AUDIT (Alcohol Use Disorders Identification Test) [dissertação]. Pelotas: Universidade Federal de Pelotas; 1999.

12. Lima CT, Freire AC, Silva AP, Teixeira RM, Farrel M, Prince M. Concurrent and construct validity of the AUDIT in an urban Brazilian sample. Alcohol Alcohol. 2005;40(6):584-9.

13. Henrique IF, Micheli D, Lacerda RB, Lacerda LA, Formigoni ML. Validação da versão brasileira do teste de triagem do envolvimento com álcool, cigarro e outras substâncias (ASSIST). Rev Assoc Med Bras. 2004;50:199-206.

14. Von der Borch P, Dimitriadis K, Störmann S, Meinel FG, Moder S, Reincke $\mathrm{M}$, et al. A novel large-scale mentoring program for medical students based on a quantitative and qualitative needs analysis. GMS Z Med Ausbild. 2011;28(2):doc26.

15. Berk RA, Berg J, Mortimer R, Walton-Moss B, Yeo TP. Measuring the effectiveness of faculty mentoring relationships. Acad Med. 2005;80(1):66-71.

16. Ministério da Saúde. Vigitel Brasil 2018: vigilância de fatores de risco e proteção para doenças crônicas por inquérito telefônico: estimativas sobre frequência e distribuição sociodemográfica de fatores de risco e proteção para doenças crônicas nas capitais dos 26 estados brasileiros e no Distrito Federal em 2018 [Internet]. Brasília: Ministério da Saúde: 2019 [acesso em: 07 jul 2020]. Disponível em: https://portalarquivos2.saude.gov.br/images/pdf/2019/julho/25/ vigitel-brasil-2018.pdf

17. Machado CS, Moura TM, Almeida RJ. Estudantes de medicina e as drogas: evidências de um grave problema. Rev Bras Educ Med. 2015;39:159-67.

18. Petroianu A, Reis DC, Cunha BD, Souza DM. Prevalência do consumo de álcool, tabaco e entorpecentes por estudantes de medicina da Universidade Federal de Minas Gerais. Rev Assoc Med Bras. 2010;56:568-71.

19. Paduani GF, Barbosa GA, Morais JC, Pereira JC, Almeida MF, Prado MM, et al. Consumo de álcool e fumo entre os estudantes da Faculdade de Medicina da Universidade Federal de Uberlândia. Rev Bras Educ Med. 2008;32:66-74.

20. Amorim AV, Kikko EO, Abrantes MM, Andrade VL. Álcool e alcoolismo: estudo de prevalência entre discentes do Curso de Medicina da Unifenas em Belo Horizonte - Minas Gerais. Rev Méd Minas Gerais. 2008;18:16-23.

21. Lameu JN, Salazar TL, Souza WF. Prevalência de sintomas de stress entre graduandos de uma Universidade Pública. Psicol Educ. 2016;42(1):13-22.

22. Correia D, Martynhak BJ, Pereira M, Siba IP, Ribeiro AF, Camarini R, et al. Reduction of ethanol intake by corticotropinreleasing factor receptor-1 antagonist in "heavy-drinking" mice in a free-choice paradigm. Psychopharmacology (Berl). 2015;232:27319.

23. Torres-Berrio A, Cuesta S, Lopez-Guzman S, Nava-Mesa MO. Interaction between stress and addiction: contributions from latinamerican neuroscience. Front Psychol. 2018;9:2639. Front Psychol

24. Trindade BP, Diniz AV, Sá AR Júnior. Uso de drogas entre estudantes universitários: uma perspectiva nacional. Rev Med Saude Brasilia. 2018;7:52-60.

25. Pinheiro MA, Torres LF, Bezerra MS, Cavalcante RC, Alencar $\mathrm{RD}$, Donato AC, et al. Prevalência e fatores associados ao consumo de álcool e tabaco entre estudantes de medicina no nordeste do Brasil. Rev Bras Educ Med. 2017;41:231-9.

26. Sterling, P. Allostasis: a model of predictive regulation. Physiol Behav. 2012;106:5-15.

27. Ganzel BL, Morris PA, Wethington E. Allostasis and the human brain: integrating models of stress from the social and life sciences. Psychol Rev. 2010;117:134-74.

28. Aguiar AS, Catelli R, Toledo L, Ubaldo L, Silva CJ, Fonseca VA. Estudo da atitude diante do paciente alcoolista e do conhecimento sobre alcoolismo em função do padrão de beber de estudantes de medicina. Rev Bras Educ Med. 2018;42:49-56.

29. Bellodi PL, Martinho T, Massaroppe B, Martins MA, Santos MA. Temas para um Programa de Tutoria em Medicina: uma investigação das necessidades dos alunos da FMUSP. Rev Bras Educ Med. 2004;28:119-27.

30. Musyoka CM, Mbwayo A, Donovan DM, Mathai M. mHealthbased peer mentoring for prevention of alcohol and substance abuse among first year university students: protocol for quasi-experimental intervention. J Subst Use. 2020;26(1):53-9.

\section{Como citar:}

Silva IS, Pilio TP, Ribeiro AF. Consumo de substâncias psicoativas pelos estudantes de medicina e sua relação com o programa de mentoria. Rev Med UFC. 2021;61(1):1-8. 